\title{
Ophiopogonin D Reduces Myocardial Ischemia-Reperfusion Injury via Upregulating CYP2J3/EETs in Rats
}

\author{
Xiaoyan Huang ${ }^{a, b} \quad$ Yuguang Wang ${ }^{b}$ Yi Wang ${ }^{a} \quad$ Liang Yang $^{b}$ Jia Wang ${ }^{b}$ \\ Yue Gao \\ aTianjin University of Traditional Chinese Medicine, Tianjin, bepartment of Pharmaceutical Sciences, \\ Beijing Institute of Radiation Medicine, Beijing, China
}

\section{Key Words}

Ophiopogonin D • Shenmai Injection • CYP2J3/EETs • PI3K/Akt/eNOS • MI/R injury

\begin{abstract}
Background/Aims: Epoxyeicosatrienoic acids (EETs) are cytochrome P450 epoxygenase (CYP) metabolites of arachidonic acid and have multiple cardiovascular effects. Ophiopogonin D (OP-D) is an important effective monomeric component in Shenmai injection (SM-I). Both have been reported to have a variety of biological functions, including anti-inflammatory, anti-oxidant, and anti-apoptotic effects. We previously demonstrated that OP-D-mediated cardioprotection involves activation of CYP2J2/3 and enhancement of circulating EETs levels in vitro and can be developed as a novel drug for the therapy of myocardial ischemia-reperfusion $(\mathrm{MI} / \mathrm{R})$ injury. We therefore hypothesized that the protective effects of OP-D and SM-I against $\mathrm{MI} / \mathrm{R}$ injury are associated with increased expression of CYP2J3 and enhanced circulating 11,12EET levels in vivo. Methods: A rat model of MI/R injury was generated by ligation of the left anterior descending coronary artery for $40 \mathrm{~min}$, followed by reperfusion for $2 \mathrm{~h}$ to determine the protective effects and potential mechanisms of OP-D and SM-I. Electrocardiogram and ultrasonic cardiogram were used to evaluate cardiac function; 2,3,5-triphenyltetrazolium chloride was used to measure myocardial infarct size; hematoxylin and eosin staining and transmission electron microscopy were used to observe the morphology of myocardial tissue; and the expression of related proteins in the mechanistic study was observed by western blot analysis. Results: We found that OP-D and SM-I exert protective effects on MI/R injury, including regulation of cardiac function, reduction of lactate dehydrogenase and creatine kinase production, attenuation of myocardial infarct size, and improvement of the recovery of damaged myocardial structures. We found that OP-D and SM-I activate CYP2J3 expression and increase levels of circulating 11,12-EET in MI/R-injured rats. Conclusion: We tested the hypothesis that the cardioprotective effects of OP-D and SM-I on MI/R injury are associated with increased expression of CYP2J3 and enhanced circulating 11,12-EET levels in rats. Taken together, our results show that the effects of OP-D and SM-I were also mediated by the




\section{Cellular Physiology Cell Physiol Biochem 2018;49:1646-1658

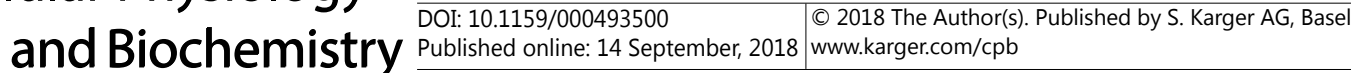 \\ Huang et al.: Ophiopogonin D Reduces Myocardial I/R Injury via CYP2J3/EETs}

activation of the PI3K/Akt/eNOS signaling pathway, while inhibition of the NF-KB signaling pathway and antioxidant and anti-apoptotic effects were involved in the cardioprotective effects of OP-D and SM-I.

(C) 2018 The Author(s)

Published by S. Karger AG, Basel

\section{Introduction}

Cardiovascular diseases (CVDs), including coronary artery disease and acute myocardial infarction, is a human health crisis that remains one of the leading causes of death worldwide [1]. Ischemic heart disease remains the leading cause of mortality and disability worldwide [2]. Although timely reperfusion therapy is the primary treatment, reperfusion itself results in major cardiac damage, commonly referred to as myocardial ischemia-reperfusion (MI/R) injury [3]. MI/R injury lacks effective treatments [4]. Epoxyeicosatrienoic acids (EETs) are metabolites of arachidonic acid (AA) generated by cytochrome P450 epoxygenases (CYPs) and are important lipid mediators involved in regulating cardiac function and protection against MI/R [5-8]. In the heart, CYP2J is abundant and predominantly involved in the biosynthesis of $5,6-, 8,9-, 11,12-$, and 14, 15-EETs from AA $[9,10]$. EETs play important roles in cellular signaling and have numerous biological activities in the cardiovascular system including vasodilatory [11], anti-inflammatory [11, 12], anti-fibrotic [13], and anti-apoptotic effects [14], which also play a significant role in the improved postischemic functional recovery in isolated hearts overexpressing CYP2J2. Thus, drugs that can increase the expression of CYP2J and subsequently increase EET concentrations are urgently needed. It is also important to explore the underlying mechanisms by which EETs exert their effects and whether increases in circulating EETs mediated by CYP2J3 overexpression can prevent MI/R injury.

Ophiopogonin D (OP-D), a steroidal glycoside isolated from the traditional Chinese herbal agent Radix Ophiopogon japonicus, has a significant protective effect on the cardiovascular system because of its pharmacological activities, such as anti-inflammatory $[15,16]$, antioxidative [17], and anti-apoptotic effects [18]. We previously demonstrated that OP-D can increase CYP2J2 and 11,12-EET production, plays a protective role as an effective antiinflammatory in angiotensin II-induced (Ang II-induced) endothelial injury [19], and can be developed as a novel drug for the therapy of MI/R injury. SM-I contains extracts from red ginseng and ophiopogonin root and has the effect of expanding peripheral blood vessels and increasing coronary blood flow [20], as well as anti-myocardial ischemia effects, and has been widely used to treat cardiac diseases in China. Our previous studies demonstrated that SM-I activates CYP2J3 expression in a dose-dependent manner, preventing myocardial hypertrophy.

We previously demonstrated that 11, 12-EET inhibits Ang II-induced inflammatory responses by repressing NF- $\kappa \mathrm{B}$ activation, and inhibits apoptosis via a caspase-dependent pathway in endothelial cells. Moreover, 11, 12-EET attenuates MI/R injury in animal models. Mechanistic studies suggested that EETs act as vasodilatory substances in several vascular beds via activation of ATP-sensitive $\mathrm{K}^{+}$channels [8], endothelial nitric oxide synthase (eNOS) [5], mitogen-activated protein kinase (MAPK) [8], and phosphatidylinositol-3 kinase (PI3K)/ Akt signaling pathways [6] following ischemia.

Thus, it is crucial to investigate the mechanism of MI/R injury to develop new treatments and preventive strategies. This study used OP-D as the typical active component of SM-I, investigating its effects on MI/R injury in rats and to deduce the underlying mechanisms. This study also examined OP-D's effects on the concentrations of EET metabolites, and evaluated its influence on the cardiac expression and activity of CYP2J3 in rats. We hypothesized that OP-D pretreatment ameliorates inflammation, oxidation stress, and apoptosis of MI/R injury by upregulating CYP2J3/EET and thus activating the PI3K/Akt/eNOS signaling pathway.

\section{Materials and Methods}

Chemicals and Reagents

OP-D (molecular weight $855.017 \mathrm{~g} / \mathrm{mol}$; purity 98\%, as determined by high-performance liquid chromatography) isolated from the traditional Chinese herbal agent Radix Ophiopogon japonicus, was 


\section{Cellular Physiology Cell Physiol Biochem 2018;49:1646-1658 \begin{tabular}{l|l|l} 
and Biochemistry Published online: 14 September, 2018 & $\begin{array}{l}\text { (c) } 2018 \text { The Author(s). Published by S. Karger AG, Basel } \\
\text { www.karger.com/cpb }\end{array}$
\end{tabular}}

Huang et al.: Ophiopogonin D Reduces Myocardial I/R Injury via CYP2J3/EETs

purchased from Shanghai Yuanye Biotechnology Co., Ltd. (Shanghai, China). SM-I (10 mg/mL radix ginseng rubra, $10 \mathrm{mg} / \mathrm{mL}$ Radix Ophiopogonis japonicus), was made by Huarun San-Jiu Pharmaceutical Co., Ltd. (Shenzhen, China). 2, 3,5-Triphenyltetrazolium chloride (TTC) and TRIzol reagent were obtained from Sigma-Aldrich Co. (St. Louis, MO). TransScript First-Step RT-PCR SuperMixFast and SYBR Green Master Mix were purchased from TransGen Biotech Co, Ltd. (Beijing, China). Creatine kinase (CK) and (LDH) reagent kits were purchased from Beisheng North Control Biotechnology Co., Ltd. (Beijing, China). The antibody against CYP2J3 was obtained from Bioss (Boston, MA). Primary antibodies against NF- $\kappa$ B p65, IкB $\alpha$, gp91, e-NOS, and phosphorylated eNOS (p-eNOS [Ser1177]) were purchased from Abcam (Cambridge, MA). Antibodies against JNK, p-JNK, and goat anti-rabbit and anti-mouse IgG-horseradish peroxidase were obtained from Santa Cruz Biotechnology, Inc. (Paso Robles, CA). SOD2, catalase, Akt, p-Akt (Thr308), and caspase-3 antibodies were from Cell Signaling Technology (Danvers, MA). The Pierce ECL Western Blotting Kit was purchased from Thermo Fisher Scientific (Grand Island, NY).

\section{Animals}

Male Sprague-Dawley rats $(240 \pm 20$ g, specific pathogen free, certification no. SCXK 2016-0002), were purchased from Beijing Sibeford Biotechnology Co. Ltd. (Beijing, China). All experimental procedures and protocols were in compliance with the Laboratory Animal Center of the Academy of Military Medical Sciences and complied with the relevant national legislation and local guidelines. Rats were sheltered in polycarbonate cages with a 12 -h light/dark cycle in a temperature-controlled $\left(22^{\circ} \mathrm{C} \pm 1{ }^{\circ} \mathrm{C}\right)$, humiditycontrolled $(60 \% \pm 5 \%)$ room. All rats were fed with standard rat chow and allowed fresh water ad libitum until the experiment started.

\section{Animal preparation}

As shown in Table 1, rats were sequentially divided into 4 groups: sham-operated, MI/R injury, OP-D, and SM-I, treated for 2 weeks. The OP-D solution was freshly prepared before use each day by dissolving in normal saline with the help of ultrasound. No organic solvent was added. The rats were injected intraperitoneally with $20 \mathrm{mg} / \mathrm{kg}$ OP-D solution every day. The SM-I dosage was calculated on based on 5 times the normal clinically used dosage $(40 \mathrm{~mL}$ per person daily, weight $=60 \mathrm{~kg})$. The rats were injected intraperitoneally with the maximum dose $(20 \mathrm{~mL} / \mathrm{kg} /$ day $)$ according to our previous study and other data $[21,22]$.

\section{Animal model of $M I / R$ injury}

Experiments were designed to investigate the cardioprotective effects of OP-D and SM-I against MI/R injury (Fig. 1). MI/R injury was evoked by ligation of the left anterior descending (LAD) coronary artery for $40 \mathrm{~min}$, followed by reperfusion for $2 \mathrm{~h}$. The rats were fasted for $12 \mathrm{~h}$ before the operation and had unlimited access to drinking water. The rats were deeply anesthetized with $10 \%$ chloral hydrate by intraperitoneal injection at a dose of $0.3 \mathrm{ml} / 100 \mathrm{~g}$, and then attached to the operating table under ether anesthesia. After routine disinfection of the precordium, the skin was incised, the chest muscles were bluntly dissected, and the chest cavity was opened between the 3rd and 4th ribs along the left border of the sternum; the pericardium was incised, and the heart was exposed. Using a needle holder to hold a small round curved needle, a 5/0-T line was placed at a distance of $2 \mathrm{~mm}$ from the lower margin of the left atrial ear in front of the descending branch of the left anterior descending coronary artery. After stabilization, a silicon gel tube $1.5 \mathrm{~mm}$ in diameter was placed between the ligation line and the blood vessel. The ligature was tightened so that the silicone tube pressed against the left ventricular branch of the coronary artery to cause occlusion. Then, the heart was put back into the chest cavity and the gas was squeezed out of the chest cavity, which was then closed followed by closure of the chest incision. The ligature was released during reperfusion and the silicone tube was then extracted. The MI/R, OP-D, and SM-I rat groups were subjected to ischemia for $40 \mathrm{~min}$

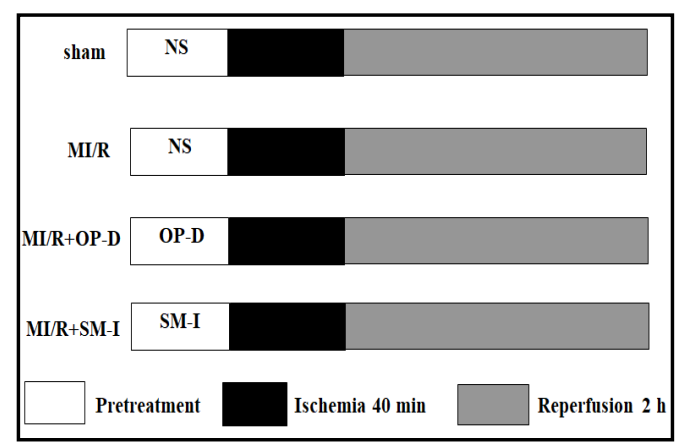

Fig. 1. Experimental protocols for ischemia and reperfusion

Table 1. Groups, numbers, and administration of OP-D and SM-I in rats

\begin{tabular}{lcc}
\hline Groups & Numbers & Treatment (14 days) \\
\hline sham & 12 & Normal saline \\
MI/R & 12 & Normal saline \\
MI/R+OP-D & 12 & $20 \mathrm{mg} / \mathrm{kg} /$ day \\
MI/R+SM-I & 12 & $10 \mathrm{~mL} / \mathrm{kg} /$ day \\
\hline
\end{tabular}




\section{Cellular Physiology Cell Physiol Biochem 2018;49:1646-1658

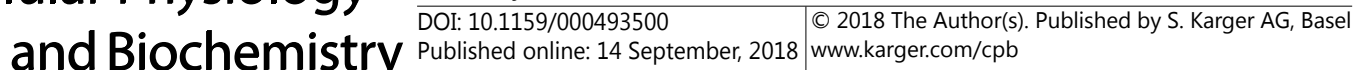

and reperfusion for $2 \mathrm{~h}$ (Fig. 1). After reperfusion, the rats were deeply anesthetized, and then a follow-up experiment was conducted.

\section{ECG and ultrasonic cardiogram}

During the whole ischemia-reperfusion period, three ECG tests were performed (pre-ischemia, $15 \mathrm{~min}$ of ischemia, and $15 \mathrm{~min}$ of reperfusion). The rats were given anesthesic in the supine position to immobilize their limbs and head. The ECG changes in the limbs were recorded with the PowerLab 8/30 (ADInstruments, New South Wales, Australia), recorded from lead II. All experimental animals were subjected to ECG recording. M-mode images of the left ventricle were obtained by ultrasonic cardiogram instrument to determine heart rate, diastolic anterior wall thickness, diastolic posterior wall thickness, percentage ejection fraction (EF), and percentage fractional shortening (FS).

\section{Serum myocardial enzyme detection}

After 40 min of myocardial ischemia and $2 \mathrm{~h}$ of reperfusion, all rats were narcotized with chloral hydrate $(0.3 \mathrm{~mL} / 100 \mathrm{~g})$. Blood samples were collected from the abdominal aorta, and serum was separated from the blood by centrifugation at $3,000 \times g$ at $4^{\circ} \mathrm{C}$ for $10 \mathrm{~min}$ and stored at $-80^{\circ} \mathrm{C}$. Serum levels of LDH and CK were measured in an automated chemical analyzer (AU1000, Olympus, Tokyo, Japan) to evaluate MI/R injury.

\section{Measurement of myocardial infarct size by TTC}

The size of the infarcted myocardium was determined through TTC staining. Briefly, after a successful $\mathrm{MI} / \mathrm{R}$ period, the LAD coronary artery was reoccluded. After the blood was obtained, the heart was removed at once and washed in cold normal saline. Then, the hearts were frozen and stored at $-20^{\circ} \mathrm{C}$ for $20 \mathrm{~min}$. After freezing, the hearts were sliced perpendicularly along the long axis from apex to base in 1- to 2-mm sections. The heart slices were incubated with 1\% TTC in phosphate-buffered solution (PBS), pH 7.4, at $37^{\circ} \mathrm{C}$ for $10-15 \mathrm{~min}$, and then fixed in a $10 \%$ formaldehyde solution. After incubation overnight in $10 \%$ formaldehyde, the non-infarcted and infarcted tissues within the anatomic area-at-risk (AAR) were separated and subsequently photographed using a digital camera. Areas of red-stained viable tissue and white unstained necrotic tissue were analyzed by ImageJ software. The infarct size of the myocardium was expressed as a percentage of the AAR.

\section{Hematoxylin and eosin (H\&E) staining}

After a 2-h reperfusion period, 5 rats were randomly selected from each group and sacrificed for histological assessment. The hearts were washed in cold normal saline and immediately put into $10 \%$ formalin solution for $48 \mathrm{~h}$. They were then embedded in paraffin, serially sectioned, and stained with H\&E.

\section{Transmission electron microscopy}

Heart tissue pieces of 1-2 $\mathrm{mm}^{3}$ removed from the ischemic AAR were fixed with $2.5 \%$ glutaraldehyde in PBS, $1 \%$ osmium, and 0.1 mole/L tetroxide in PBS, dehydrated with an acetone gradient, embedded with EPON-812 epoxy resin in a warm box at $45^{\circ} \mathrm{C}$ overnight, and polymerized at $60^{\circ} \mathrm{C}$ for $48 \mathrm{~h}$. Ultrathin sections (60-70 $\mathrm{nm}$ thick) were placed on grids (200 mesh) under a light microscope, double staining of uranyl acetate and lead citrate was carried out, ultrastructural changes of the myocardium were observed under a transmission electron microscope (H-7650, Hitachi, Tokyo, Japan), and photographs were taken.

Detection of 11, 12-dihydroxyeicosatrienoic acid (11, 12-DHET)

EETs are unstable and can be enzymatically hydrated to the corresponding stable metabolite DHETs by soluble epoxide hydrolases (sEHs). To evaluate the in vivo EET level, an enzyme-linked immunosorbent assay (ELISA) kit (Detroit R\&D, Detroit, MI) was used to determine the concentration of 11, 12-DHET. ELISAs were performed according to the manufacturer's instructions.

\section{Western blot analysis}

Heart tissues were harvested and lysed in lysis buffer containing protease inhibitors and phosphatase inhibitors. The protein concentrations were determined by using a BCA protein assay kit (Kangwei Century, Beijing, China). Total protein samples were separated by $10 \%$ sodium dodecyl sulfate polyacrylamide gel electrophoresis and then transferred to nitrocellulose membranes (Millipore, Temecula, CA). The membranes were blocked with 5\% nonfat milk dissolved in PBS for at least $3 \mathrm{~h}$. Membranes were incubated overnight at $4^{\circ} \mathrm{C}$ with primary antibodies against CYP2J3, Akt, p-Akt (Thr308), eNOS, p-eNOS (Ser1177), NF - $\kappa \mathrm{B}$ p65, IкB $\alpha, \mathrm{p}-\mathrm{I} \kappa \mathrm{B} \alpha$ (Ser32), gp91, SOD2, catalase, JNK, p-JNK, and caspase-3. Then the membranes were incubated with secondary antibodies for $1 \mathrm{~h}$ at room temperature. Immunoreactive bands were revealed 


\section{Cellular Physiology Cell Physiol Biochem 2018;49:1646-1658

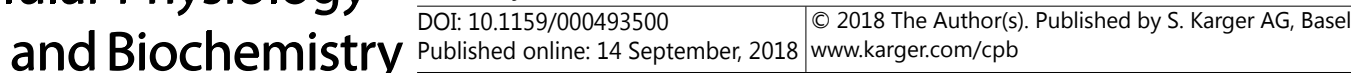 \\ Huang et al.: Ophiopogonin D Reduces Myocardial I/R Injury via CYP2J3/EETs}

with Super Signal West Femto Maximum Sensitivity Substrate (Kangwei Century) and visualized with ImageQuant LAS 500 (Healthcare Bio-Sciences AB, Uppsala, Sweden).

RNA isolation and quantitative real-time PCR

We extracted total RNA from heart tissues using TRIzol reagent and then reverse transcribed it into cDNA. Real-time PCR reactions were performed in TransScript SYBR Green Master Mix on an ABI Prism 7500 real-time PCR instrument (Applied Biosystems, Foster City, CA, USA). Each group contained 5 samples and was repeated three times. The relative amount of each gene was normalized to the amount of GAPDH. The primer sequences are provided in Table 2.

Statistical analysis

All data are expressed as mean \pm standard deviation. Comparisons between groups were performed by a one-way analysis of variance, followed by Bonferroni's post hoc test. Differences with $P<0.05$ were considered statistically significant.

\section{Results}

OP-D improves cardiac function in the reperfused heart

The ST segment changes in ECG lead II induced by MI/R injury in rats were inhibited by pretreatment with OP-D and SM-I. The changes in ECG in the limbs after $15 \mathrm{~min}$ of reperfusion were recorded. In our ECG study, 6 rats were randomly selected from each group. The experiment showed that the ST segment of lead II in rats was significantly higher than $0.1 \mathrm{mV}$ or the $\mathrm{T}$ wave, which was used as a criterion for the success of the ligation model of the anterior descending branch. As depicted in Fig. $2 \mathrm{~A}$, the ST segment of ECG lead II was obviously elevated in the heart following MI/R, a phenomenon that was reversed by OP-D and SM-I.

OP-D and SM-I improved cardiac performance of the infarcted hearts. The cardiac function of rats in the different groups was evaluated by echocardiography at $2 \mathrm{~h}$ after LAD artery surgery, and the left ventricular (LV) FS and EF were calculated. As indicated in Fig. $2 \mathrm{~B}$, the LV structure clearly changed and its function was weakened in the MI/R group. In contrast, OP-D and SM-I remarkably improved LV function induced by MI/R, and the LV motion amplitude of SM-I was better than that in the OP-D group. Ultrasonic detection suggested a marked improvement of cardiac function in the OP-D and SM-I groups in the context of EF and FS parameters, while the MI/R group prominently decreased (Table 3).

Table 2. Primer sequences used for real-time PCR

\begin{tabular}{lcc}
\hline Gene & Forward primer (5'-3') & Reverse primer (5'-3') \\
\hline CYP2J3 & CATTGAGCTCACAAGTGGCTTT & CAATTCCTAGGCTGTGATGTCG \\
GAPDH & GGCCTCCAAGGAGTAAGACC & AGGGGAGATTCAGTGTGGTG \\
\hline
\end{tabular}

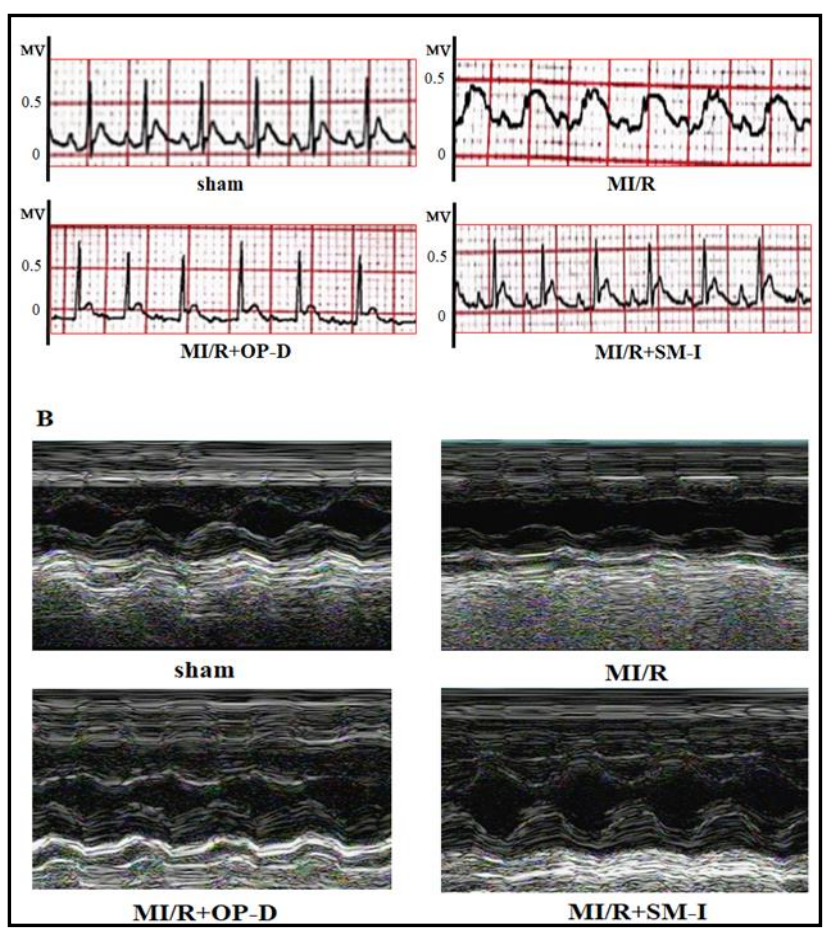

Fig. 2. Effect of OP-D and SM-I on cardiac function after MI/R injury. (A) A typical electrocardiogram recorded after 15-min reperfusion. (B) Representative echocardiographic images of hearts from the different groups $(n=6)$. 


\section{Cellular Physiology Cell Physiol Biochem 2018;49:1646-1658

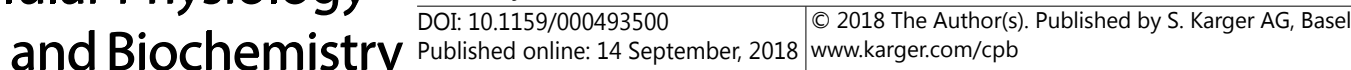 \\ Huang et al.: Ophiopogonin D Reduces Myocardial I/R Injury via CYP2J3/EETs}

\section{OP-D pretreatment protected MI/R injury and ameliorated infarct area in the reperfused}

heart

As it is well known that serum LDH and CK are sensitive markers for myocardial injury, we analyzed LDH and CK serum levels and the infarct size, comparing the effects of OP-D with those of SM-I pretreatment on MI/R injury. As shown in Table 4, the serum levels of LDH and CK increased dramatically in the MI/R group. OP-D and SM-I pretreatment reduced the increase in LDH and CK levels after MI/R injury. Furthermore, the levels were significantly lower in the OP-D group compared with the SM-I group.

TTC staining was used to detect the infarct volume $2 \mathrm{~h}$ after reperfusion. As observed directly, extensive infarction was developed in the left ventricle in the MI/R group compared with the sham group; SM-I treatment markedly reduced the infarction compared with OP-D (Fig. 3A). Through quantitative analysis, the infarct volume percentage of the ischemic area was significantly higher in the MI/R group compared with the sham group $(47.67 \% \pm 3.79 \%$ vs. $0.00 \pm$ 0.00 , respectively, Fig. 3B). However, OP-D and SM-I treatment significantly reduced the infarct volume from $47.67 \% \pm 3.79 \%$ to $30.67 \% \pm 3.06 \%$ and $20.33 \%$ $\pm 2.08 \%$, respectively, compared with the MI/R group (Fig. 3B). These results demonstrate that OP-D and SM-I reduced the percentage of the infarct area.

\section{Effects of OP-D and SM-I on heart histopathology} and myocardial ultrastructure.

In histopathological evaluation, as shown in Fig. 4A, we found that in the sham group the cardiac muscle fibers were intact, arranged in order, the structure was relatively clear, with no necrosis or infiltration of inflammatory cells. In contrast, a large number of infiltrated neutrophils, myocardial fiber rupture, and necrosis of myocardial cells in the infarctrelated area were observed in the MI/R group. However, we observed that OP-D and SM-I treatment in MI/R rats alleviated the myocardial fiber damage and the occurrence of inflammatory reactions.

To elucidate the effect of OP-D and SM-I on myocardial cell ultrastructure, transmission electron microscopy was performed (Fig. 4B). We observed that in the sham group the arrangement of myocardial fibers was uniform, the structure of myocardial mitochondria was intact, and the nuclei were clear. In contrast, in the MI/R group, the arrangement of myocardial fibers was disordered, the myocardial mitochondrial damage was obvious, and myocardial cell nuclei were vacuolated or even dissolved. However, OP-D and SM-I pretreatment markedly relieved the ultrastructural changes compared with the MI/R model group, suggesting that it may be associated with anti-mitochondrial damage.

Table 4. Effects of OP-D and SM-I on serum $\mathrm{LDH}$ and $\mathrm{CK}$ in rats with $\mathrm{MI} / \mathrm{R}$ injury. $n=6$; ${ }^{* *} P<0.01,{ }^{* * *} P<0.001$ versus sham group; ${ }^{\#} P<0.05,{ }^{\# \#} P<0.01$ versus MI/R group

\begin{tabular}{lcc}
\hline Groups & LDH (U/L) & CK (U/L) \\
\hline sham & $245 \pm 20$ & $1,109 \pm 98$ \\
MI/R & $918 \pm 72 * *$ & $3,849 \pm 234^{* * *}$ \\
MI/R+OP-D & $309 \pm 52^{\# \#}$ & $1,016 \pm 253^{\# \#}$ \\
MI/R+SM-I & $595 \pm 14^{\#}$ & $1,843 \pm 139^{\#}$ \\
\hline
\end{tabular}

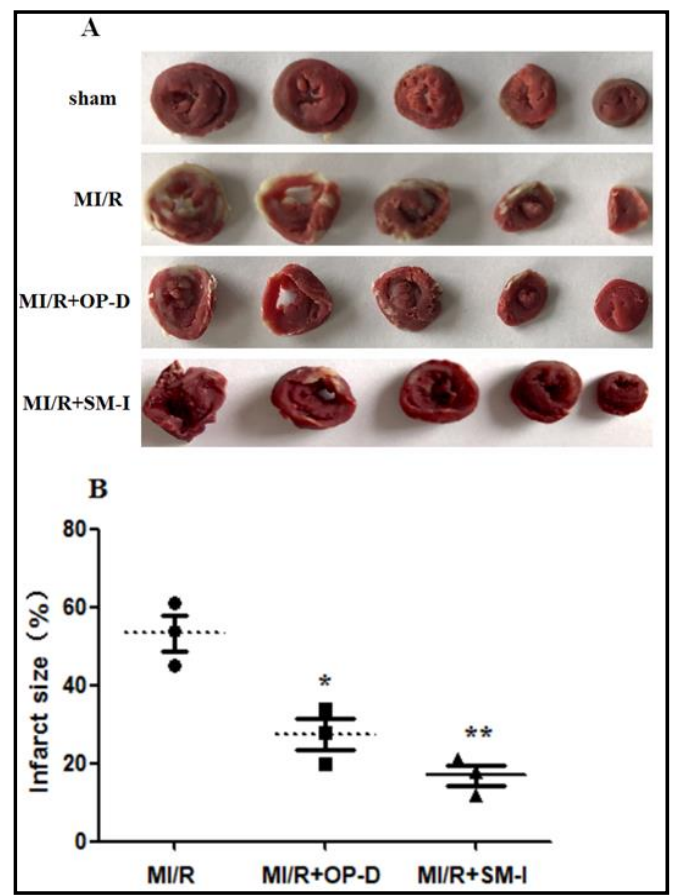

Fig. 3. Effects of OP-D and SM-I on the myocardial infarct size of cardiac tissue. (A) Representative images of infarcted heart muscle from the different groups. (B) Representative data showing the infarct volume percentage of myocardial tissue ( $\mathrm{n}$ $=6{ }^{*} P<0.05,{ }^{* *} P<0.01$ versus MI/R group). 


\section{Cellular Physiology Cell Physiol Biochem 2018;49:1646-1658

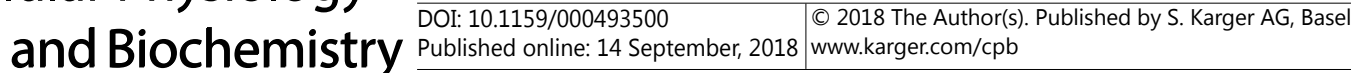

OP-D and SM-I pretreatment attenuated cardiomyocyte inflammation, oxidative stress, and apoptosis during $M I / R$ injury in rats

To further investigate the molecular mechanisms underlying the antiinflammatory, anti-oxidative, and antiapoptotic effects of OP-D and SM-I on MI/R injury, western blot analysis of Akt, eNOS,

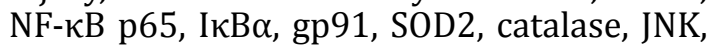
and caspase- 3 proteins was performed. As indicated in Fig. 5A-E, the protein level of NF$\kappa \mathrm{B}$ p65 in the nucleus, p-IкB $\alpha$, gp91, p-JNK, and caspase- 3 was significantly increased in the heart tissues following MI/R injury, a phenomenon that was reversed by OP-D and SM-I pretreatment, while the protein level of p-Akt, p-eNOS, I $\kappa \alpha$, SOD2, and catalase was remarkably increased in the OP-D and SM-I groups when compared to the MI/R group. Together, these data suggested that OP-D and SM-I have anti-inflammatory, anti-oxidative, and anti-apoptotic effects in MI/R injury.

OP-D activated CYP2J3 expression and increased serum and tissue production of 11, 12-DHET during reperfusion

Accumulating evidence suggests that CYP2J2 overexpression and exogenous 11, 12 -EET may serve a protective mechanism in the ischemic myocardium and various CVDs $[23,24]$. Our previous studies demonstrated that OP-D and exogenous 11, 12-EET play protective roles as anti-inflammatory and anti-apoptotic agents following Ang IIinduced endothelial injury via activating CYP2J2 and increasing 11, 12-EET production in human umbilical vein endothelial cells (HUVECs) [19, 25]. Based on these findings, we tested the hypothesis that OP-D and SM-I exert a protective role in MI/R injury and are associated with increased cardiac expression of CYP2J3 and enhanced circulating 11, 12-EET levels in rats. Therefore, in the current study, we analyzed CYP2J3 protein and mRNA expression and concentrations of 11, 12DHET. The heart is the major site of EET-producing CYP2J2, which is reduced during MI/R injury. Our study showed that CYP2J3 protein and mRNA expression was enhanced by OP-D and SM-I administration relative to that in the MI/R group (Fig. 6A-C). OP-D and SM-I also could improve the circulating and myocardial tissue 11, 12-DHET levels (Fig. 6D). Our data are consistent with the idea that enhanced cardiac CYP2J2 activity may result in increased circulating 11, 12-EET levels.

\section{Discussion}

In the current study, we demonstrated that OP-D and SM-I improved cardiac function comprehensively, and exerted anti-inflammatory, anti-oxidative stress, and anti-apoptotic effects that contribute to tissue protection under MI/R injury conditions in vivo. The mechanism underlying this protection, at least in part, involved the important role of CYP2J3 overexpression and increased levels of 11, 12-EET signaling pathways. Here, we have demonstrated that OP-D-mediated and SM-I-mediated CYP2J3 activation led to an abundant myocardial expression of CYP2J3 and elevated the levels of circulating 11, 12-EET. 


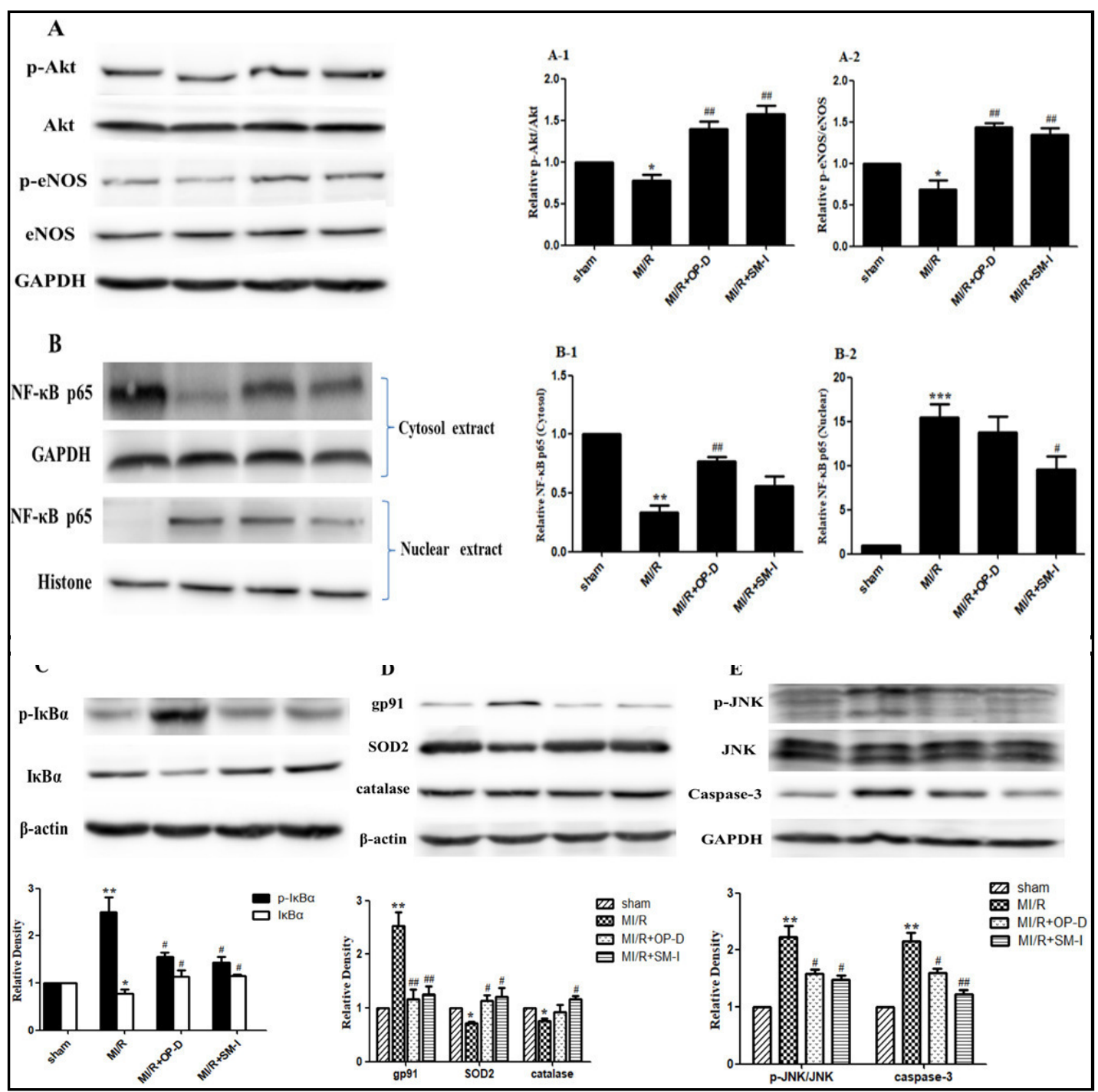

Fig. 5. Effects of OP-D and SM-I on MI/R injury. Representative western blots of (A) p-Akt, Akt, p-eNOS, and eNOS ( $\mathrm{n}=6$ for each experiment; ${ }^{*} P<0.05$ versus sham group; ${ }^{\# \#} P<0.01$ versus MI/R group). (B and C) NF-

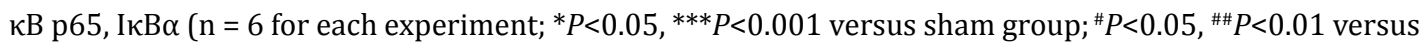
MI/R group). (D) gp91, SOD2, and catalase ( $\mathrm{n}=6$ for each experiment; ${ }^{*} P<0.05,{ }^{* *} P<0.01$ versus sham group; ${ }^{\#} P<0.05,{ }^{\# \#} P<0.01$ versus MI/R group). (E) p-JNK, JNK, and caspase- 3 in the different groups (n $=6$ for each experiment; ${ }^{* *} P<0.01$ versus sham group; ${ }^{\#} P<0.05$, ${ }^{\#} P<0.01$ versus $\mathrm{MI} / \mathrm{R}$ group).

CYP2J2, which metabolizes AA to four regioisomeric EETs, namely, 5, 6-, 8, 9-, 11, 12-, and 14, 15-EET, is widely expressed in human cardiomyocytes and endothelial cells $[26,27]$. sEH hydrolyzes EETs to less biologically active DHETs. Increased EET biosynthesis in various CVDs and during cardiac I/R suggests the hypothesis that EETs may serve a protective mechanism in the ischemic myocardium [28-32]. Both EETs and DHETs have potent vasodilatory effects in the coronary circulation. In our earlier studies, we described for the first time the activation of CYP2J3 by OP-D in cultured H9c2 cells. We demonstrated that OP-D suppressed drug-induced endoplasmic reticulum stress by upregulating the levels of CYP2J3/EET in cardiomyocytes [18]. Additionally, we found that OP-D exerts endotheliumprotective effects via the CYP2J2/EET/PPAR $\alpha$ signaling pathway in HUVECs [19]. Based on our previous studies, we propose that OP-D-induced and SM-I-induced CYP2J3 overexpression and production of circulating 11,12-EET contribute to protection from MI/R injury. Thus, our data direct attention to possibly similar mechanisms in MI/R injury.

\section{KARGER}




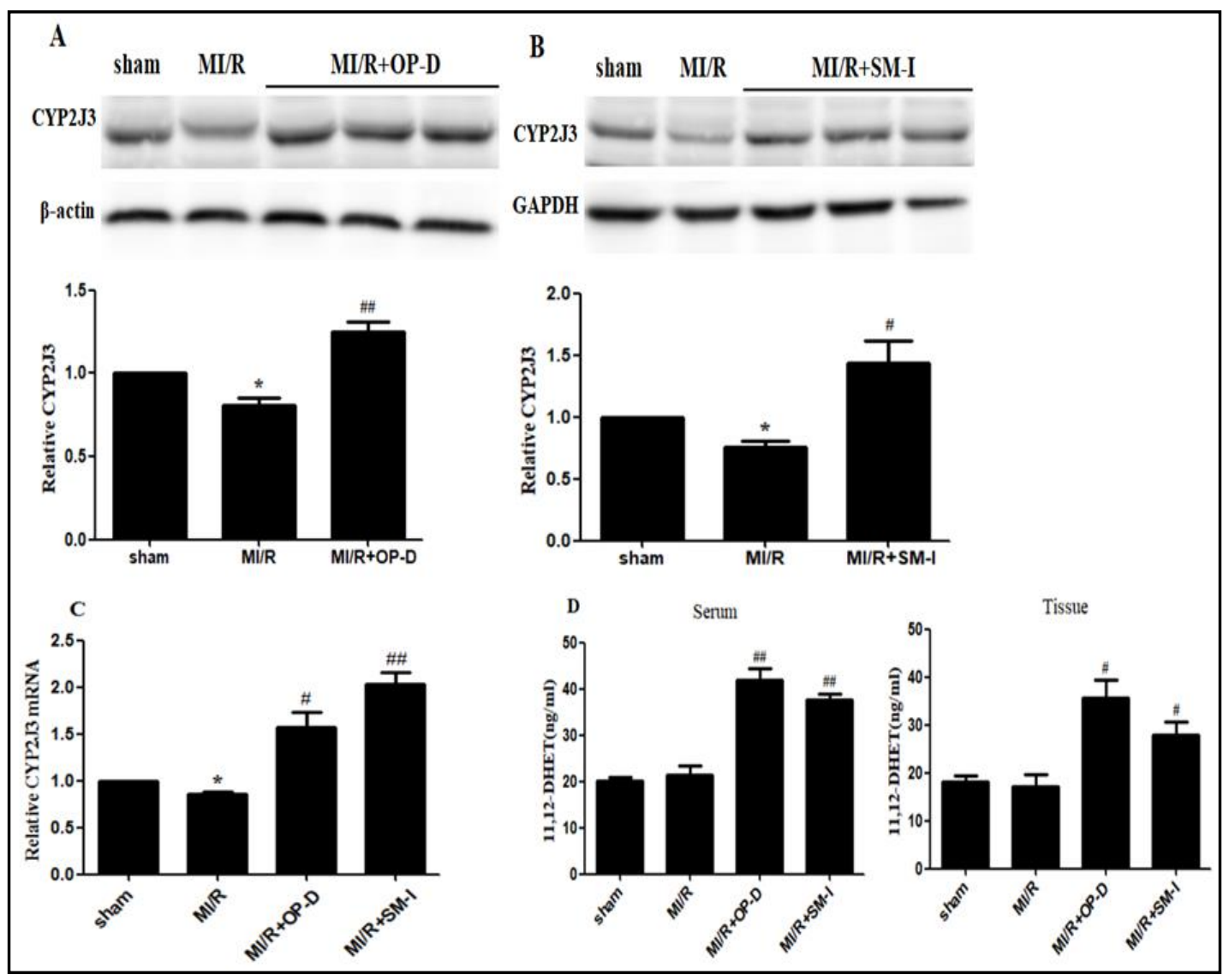

Fig. 6. Effect of OP-D and SM-I on expression of CYP2J3 and levels of 11,12-DHET in heart tissues following $\mathrm{MI} / \mathrm{R}$ injury. (A and B) Western blot results confirmed upregulation of CYP2J3 by OP-D and SM-I stimulation ( $\mathrm{n}=6$ for each experiment; ${ }^{*} P<0.05$ versus sham group; ${ }^{\#} P<0.05$, ${ }^{\#} P<0.01$ versus MI/R group). (C) CYP2J3 expression in MI/R injury is shown at the mRNA level $\left(\mathrm{n}=6\right.$ for each experiment; ${ }^{*} P<0.05$ versus sham group; ${ }^{\# P}<0.05,{ }^{\# \#} P<0.01$ versus MI/R group). (D) 11,12-DHET levels were detected in the serum and heart tissue of rats in the different groups ( $\mathrm{n}=6$ for each experiment; ${ }^{\#} P<0.05,{ }^{\# \#} P<0.01$ versus MI/R group).

The present work demonstrates a comprehensive and holistic approach to explicate the mechanism of the cardioprotective role of OP-D and SM-I based on ECG echocardiogram, biochemical analysis, histology, and infarct size. MI/R injuries dramatically cause hemodynamic changes and lead to increased LDH and CK and pathological changes and arrhythmias, all of which were reversed by pretreatment with OP-D and SM-I. Furthermore, our study shows that OP-D and SM-I attenuated mitochondrial impairment and infarct volume percentage of the ischemic area induced by MI/R injuries in rats.

Based on the wide cardiovascular protective effects of OP-D and SM-I, we further investigated the effects of MI/R injury and its possible mechanisms. We propose that OP-D and SM-I enhanced myocardial CYP2J3 activity, resulting in increased circulating 11,12-EET levels, which contributes to the protection of myocardium from I/R injury. The pathogenesis of MI/R injury consists of many mechanisms. The PI3K/Akt pathway is one of the most potent intracellular mechanisms to promote cell survival. Other studies have shown that enhanced activation of PI3K/Akt signaling was involved in the cardioprotective effects of EETs observed in MI/R-injured rats [6, 33, 34]. We hypothesized that the cardioprotective effect of OP-D and SM-I might involve the activation of the PI3K/Akt pathway. The results showed that, compared with the sham group, the changes in 11,12-DHET in serum and myocardial tissue were not significant in the MI/R group; this may be related to the physiological compensatory period of the rats. However, OP-D and SM-I pretreatment markedly activated CYP2J3 expression, enhanced Akt phosphorylation, and increased the concentration of 11, 12-DHET in the pathological condition of MI/R injury. Our data are consistent with the idea 
that CYP2J3 overexpression can cause high levels of circulating 11,12-DHET, and thus EETs increasing PI3K/Akt pathway activity. We demonstrated that these protective effects were mediated, at least in part, via the CYP2J3/EET/PI3K/Akt signaling pathway. For a more detailed mechanistic analysis, further studies using cell experiments in vitro are needed.

The notion that EETs activate eNOS via phosphorylation is supported by previous studies in a number of biological systems $[5,35,36]$. MI/R injury is reduced in mice overexpressing eNOS, suggesting cardioprotection by eNOS [37-39]. We therefore determined whether the cardioprotective effects of OP-D and SM-I in rat heart following MI/R injury are mediated by the activation of eNOS. Our results showed that OP-D and SM-I could attenuate MI/R injury by upregulating eNOS via its phosphorylation, indicating an increase in eNOS activity in MI/ R-injured rats. Many studies have reported that the PI3K/Akt/eNOS signaling pathway plays a role in MI/R damage [40,41]. The results of the present study support our hypothesis that the cardioprotective effects of OP-D and SM-I in rats can be attributed to their ability to increase the phosphorylation of eNOS through the PI3K/Akt pathway, but more data are needed to confirm the idea.

Regarding the essential role of inflammation during MI/R injury development, NF-кB is considered to be one of the most important mediators throughout the whole process of MI/R injury [42]. Our present study demonstrates that OP-D and SM-I ameliorate the inflammatory effects of MI/R injury in rats by reversing both the MI/R-induced increase in

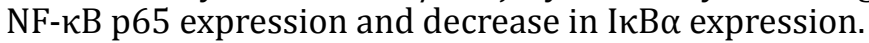

$\mathrm{MI} / \mathrm{R}$ injury is a complex pathophysiologic process [43], in which oxidative stress is a major factor and apoptosis plays a crucial role in the development of heart dysfunction following MI/R injury [44-46]. Thus, our study indicated that OP-D and SM-I pretreatment significantly attenuated oxidative stress and apoptosis via regulating the expression of prooxidative protein (gp91), anti-oxidative protein (SOD2, catalase), apoptosis-related protein JNK, p-JNK, and the activity of caspase-3 in the rats following MI/R injury.

\section{Conclusion}

In summary, we demonstrated that OP-D and SM-I protect against MI/R injury by inhibiting inflammation, oxidative stress, and apoptosis. The mechanism underlying the protective effects of OP-D and SM-I in MI/R injury may involve, at least in part, activation of CYP2J3 and upregulation of 11,12-EET in the circulation. Moreover, our data suggest that the protective mechanism involved the PI3K/Akt/eNOS signaling pathway, and crosstalk may occur between the members of this pathway in alleviating the heart damage after MI/R injury. Taken together, the results of the current study and previous data indicate that OP-D-induced CYP2J2 overexpression and increased concentration of 11, 12-EET improved cardiovascular function in vivo and in vitro via several mechanisms. However, elucidation of the mechanisms concerning OP-D-mediated upregulation of CYP2J2/3 expression requires further study. Pregnane X receptor (PXR) is an important transcriptional regulator of CYP2 enzymes and transporters in cardiovascular therapy $[47,48]$. Previously, we have found that many Chinese medicine monomers mediate protection against several diseases via activating PXR [49-51], and that OP-D induces PXR nuclear translocation. Therefore, we hypothesize that OP-D has an effect on CYP2J2/3 through PXR activation. Future studies incorporating reporter genes, siRNA, and CHIP-sequencing should elucidate the effects and complicated protective mechanisms of OP-D and SM-I.

\section{Acknowledgements}

This study was funded by the National Key Basic Research and Development Program (973 Program) (No. 2012CB518402).

\section{Disclosure Statement}

The authors have no conflict of interests to declare. 


\section{Cellular Physiology Cell Physiol Biochem 2018;49:1646-1658 \begin{tabular}{l|l|l} 
and Biochemistry 10.1159/000493500 & $\begin{array}{l}\text { C } 2018 \text { The Author(s). Published by S. Karger AG, Basel } \\
\text { www.karger.com/cpb }\end{array}$ \\
\hline
\end{tabular}}

\section{References}

1 Manuel DG, Luo W, Ugnat AM, Mao Y: Cause-deleted health-adjusted life expectancy of Canadians with selected chronic conditions. Chronic Dis Can 2003;24:108-115.

2 Keeley EC, Boura JA, Grines CL: Primary angioplasty versus intravenous thrombolytic therapy for acute myocardial infarction: a quantitative review of 23 randomised trials. Lancet 2003;361:13-20.

-3 Perrelli MG, Pagliaro P, Penna C: Ischemia/reperfusion injury and cardioprotective mechanisms: Role of mitochondria and reactive oxygen species. World J Cardiol 2011;3:186-200.

4 Xu J, Tang Y, Bei Y, Ding S, Che L, Yao J, Wang H, Lv D, Xiao J: miR-19b attenuates $\mathrm{H}_{2} \mathrm{O}_{2}$-induced apoptosis in rat H9C2 cardiomyocytes via targeting PTEN. Oncotarget 2016;7:10870-10878.

5 Gross GJ, Hsu A, Pfeiffer AW, Nithipatikom K: Roles of endothelial nitric oxide synthase (eNOS) and mitochondrial permeability transition pore (MPTP) in epoxyeicosatrienoic acid (EET)-induced cardioprotection against infarction in intact rat hearts. J Mol Cell Cardiol 2013;59:20-29.

6 Dhanasekaran A, Gruenloh SK, Buonaccorsi JN, Zhang R, Gross GJ, Falck JR, Patel PK, Jacobs ER, Medhora M: Multiple antiapoptotic targets of the PI3K/Akt survival pathway are activated by epoxyeicosatrienoic acids to protect cardiomyocytes from hypoxia/anoxia. Am J Physiol Heart Circ Physiol 2008;294:H724-735.

7 Gross GJ, Hsu A, Falck JR, Nithipatikom K: Mechanisms by which epoxyeicosatrienoic acids (EETs) elicit cardioprotection in rat hearts. J Mol Cell Cardiol 2007;42:687-691.

-8 Seubert J, Yang B, Bradbury JA, Graves J, Degraff LM, Gabel S, Gooch R, Foley J, Newman J, Mao L, Rockman HA, Hammock BD, Murphy E, Zeldin DC: Enhanced postischemic functional recovery in CYP2J2 transgenic hearts involves mitochondrial ATP-sensitive K+ channels and p42/p44 MAPK pathway. Circ Res 2004;95:506-514.

-9 Herse F, Lamarca B, Hubel CA, Kaartokallio T, Lokki AI, Ekholm E, Laivuori H, Gauster M, Huppertz B, Sugulle M, Ryan MJ, Novotny S, Brewer J, Park JK, Kacik M, Hoyer J, Verlohren S, Wallukat G, Rothe M, Luft FC, et al.: Cytochrome P450 subfamily 2J polypeptide 2 expression and circulating epoxyeicosatrienoic metabolites in preeclampsia. Circulation 2012;126:2990-2999.

10 Chen W, Yang S, Ping W, Fu X, Xu Q, Wang J: CYP2J2 and EETs protect against lung ischemia/reperfusion injury via anti-inflammatory effects in vivo and in vitro. Cell Physiol Biochem 2015;35:2043-2054.

11 Zhou C, Huang J, Chen J, Lai J, Zhu F, Xu X, Wang DW: CYP2J2-Derived EETs Attenuated Angiotensin IIInduced Adventitial Remodeling via Reduced Inflammatory Response. Cell Physiol Biochem 2016;39:721739.

12 Cai Z, Zhao G, Yan J, Liu W, Feng W, Ma B, Yang L, Wang JA, Tu L, Wang DW: CYP2J2 overexpression increases EETs and protects against angiotensin II-induced abdominal aortic aneurysm in mice. J Lipid Res 2013;54:1448-1456.

13 He Z, Yang Y, Wen Z, Chen C, Xu X, Zhu Y, Wang Y, Wang DW: CYP2J2 metabolites, epoxyeicosatrienoic acids, attenuate Ang II-induced cardiac fibrotic response by targeting Galpha12/13. J Lipid Res 2017;58:13381353.

14 Wang X, Ni L, Yang L, Duan Q Chen C, Edin ML, Zeldin DC, Wang DW: CYP2J2-derived epoxyeicosatrienoic acids suppress endoplasmic reticulum stress in heart failure. Mol Pharmacol 2014;85:105-115.

15 Qian J, Jiang F, Wang B, Yu Y, Zhang X, Yin Z, Liu C: Ophiopogonin D prevents $\mathrm{H}_{2} \mathrm{O}_{2}$-induced injury in primary human umbilical vein endothelial cells. J Ethnopharmacol 2010;128:438-445.

16 Jiang M, Kang L, Wang Y, Zhao X, Liu X, Xu L, Li Z: A metabonomic study of cardioprotection of ginsenosides, schizandrin, and ophiopogonin D against acute myocardial infarction in rats. BMC Complement Altern Med 2014;14:350.

17 Huang Q Gao B, Wang L, Zhang HY, Li XJ, Shi J, Wang Z, Zhang JK, Yang L, Luo ZJ, Liu J: Ophiopogonin D: A new herbal agent against osteoporosis. Bone 2015;74:18-28.

18 You WT, Zhou T, Ma ZC, Liang QD, Xiao CR, Tang XL, Tan HL, Zhang BL, Wang YG, Gao Y: Ophiopogonin D maintains $\mathrm{Ca} 2+$ homeostasis in rat cardiomyocytes in vitro by upregulating CYP2J3/EETs and suppressing ER stress. Acta Pharmacol Sin 2016;37:368-381.

19 Huang X, Wang Y, Zhang Z, Wang Y, Chen X, Wang Y, Gao Y: Ophiopogonin D and EETs ameliorate Ang IIinduced inflammatory responses via activating PPARalpha in HUVECs. Biochem Biophys Res Commun 2017;490:123-133.

20 Liu Q, Wu H, Wang J, Li XM: Effects of Shenmai injection on the values of CO, SV, and EF in patients undergoing off-pump coronary artery bypass graft: A randomized, clinical trial. Medicine (Baltimore) 2018;97:e0085.

21 Zhao L, Wu J, Zhang X, Kuang H, Guo Y, Ma L: The effect of Shenmai injection on the proliferation of Rat airway smooth muscle cells in asthma and underlying mechanism. BMC Complement Altern Med 2013;13:221. 


\section{Cellular Physiology Cell Physiol Biochem 2018;49:1646-1658

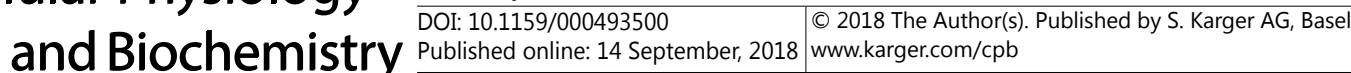 \\ Huang et al.: Ophiopogonin D Reduces Myocardial I/R Injury via CYP2J3/EETs}

22 Yu J, Xin YF, Gu LQ, Gao HY, Xia LJ, You ZQ, Xie F, Ma ZF, Wang Z, Xuan YX: One-month toxicokinetic study of SHENMAI injection in rats. J Ethnopharmacol 2014;154:391-399.

23 Potente M, Fisslthaler B, Busse R, Fleming I: 11, 12-Epoxyeicosatrienoic acid-induced inhibition of FOXO factors promotes endothelial proliferation by down-regulating p27Kip1. J Biol Chem 2003;278:2961929625.

-24 Nithipatikom K, DiCamelli RF, Kohler S, Gumina RJ, Falck JR, Campbell WB, Gross GJ: Determination of cytochrome $\mathrm{P} 450$ metabolites of arachidonic acid in coronary venous plasma during ischemia and reperfusion in dogs. Anal Biochem 2001;292:115-124.

25 Wang Y, Huang X, Ma Z, Wang Y, Chen X, Gao Y: Ophiopogonin D alleviates cardiac hypertrophy in rat by upregulating CYP2J3 in vitro and suppressing inflammation in vivo. Biochem Biophys Res Commun 2018; DOI:10.1016/j.bbrc.2018.06.110.

Zeldin DC: Epoxygenase pathways of arachidonic acid metabolism. J Biol Chem 2001;276:36059-36062.

Askari A, Thomson SJ, Edin ML, Zeldin DC, Bishop-Bailey D: Roles of the epoxygenase CYP2J2 in the endothelium. Prostaglandins Other Lipid Mediat 2013;107:56-63.

28 Gross GJ, Falck JR, Gross ER, Isbell M, Moore J, Nithipatikom K: Cytochrome P450 and arachidonic acid metabolites: role in myocardial ischemia/reperfusion injury revisited. Cardiovasc Res 2005;68:18-25.

-29 Seubert JM, Zeldin DC, Nithipatikom K, Gross GJ: Role of epoxyeicosatrienoic acids in protecting the myocardium following ischemia/reperfusion injury. Prostaglandins Other Lipid Mediat 2007;82:50-59.

-30 Xu M, Hao H, Jiang L, Wei Y, Zhou F, Sun J, Zhang J, Ji H, Wang G, Ju W, Li P: Cardiotonic Pill Reduces Myocardial Ischemia-Reperfusion Injury via Increasing EET Concentrations in Rats. Drug Metab Dispos 2016;44:878-887.

-31 Wang YX, Lu LQ, Wang XY, Mu J, Zeng XJ, Zhang LK, Tang CS, Hao G: Protective effects of 11, 12-epoxyeicosatrienoic acid preconditioning and postconditioning on myocardial ischemia/reperfusion injury in rats. Sheng Li Xue Bao 2008;60:23-28.

32 Wang YX, Zeng XJ, Lu LQ Ma LQ Jiang DQ, Mu J, Wang XY, Zhang LK, Tang CS, Hao G: Effects of 11, 12-epoxyeicosatrienoic acid preconditioning and postconditioning on $\mathrm{Ca}^{2+}$ - handling proteins in myocardial ischemia/reperfusion injury in rats. Zhongguo Yi Xue Ke Xue Yuan Xue Bao 2007;29:787-791.

33 Bodiga S, Zhang R, Jacobs DE, Larsen BT, Tampo A, Manthati VL, Kwok WM, Zeldin DC, Falck JR, Gutterman DD, Jacobs ER, Medhora MM: Protective actions of epoxyeicosatrienoic acid: dual targeting of cardiovascular PI3K and KATP channels. J Mol Cell Cardiol 2009;46:978-988.

-34 Wang Y, Wei X, Xiao X, Hui R, Card JW, Carey MA, Wang DW, Zeldin DC: Arachidonic acid epoxygenase metabolites stimulate endothelial cell growth and angiogenesis via mitogen-activated protein kinase and phosphatidylinositol 3-kinase/Akt signaling pathways. J Pharmacol Exp Ther 2005;314:522-532.

-35 Jiang JG, Chen RJ, Xiao B, Yang S, Wang JN, Wang Y, Cowart LA, Xiao X, Wang DW, Xia Y: Regulation of endothelial nitric-oxide synthase activity through phosphorylation in response to epoxyeicosatrienoic acids. Prostaglandins Other Lipid Mediat 2007;82:162-174.

-36 Qiu XW, Wang W, Jiang DQ, Wang HX, Yan L, Wang XY, Ma LQ, Lu LQ, Tang CS, Zhang LK: Effect of 11, 12-epoxyeicosatrienoic acids on hypoxia/reoxygenation injury in the human umbilical vein endothelial cells. Zhongguo Yi Xue Ke Xue Yuan Xue Bao 2006;28:803-807.

37 Wu Y, Xia ZY, Meng QT, Zhu J, Lei S, Xu J, Dou J: Shen-Fu injection preconditioning inhibits myocardial ischemia-reperfusion injury in diabetic rats: activation of eNOS via the PI3K/Akt pathway. J Biomed Biotechnol 2011;2011:384627.

-38 Li XD, Yang YJ, Geng YJ, Jin C, Hu FH, Zhao JL, Zhang HT, Cheng YT, Qian HY, Wang LL, Zhang BJ, Wu YL: Tongxinluo reduces myocardial no-reflow and ischemia-reperfusion injury by stimulating the phosphorylation of eNOS via the PKA pathway. Am J Physiol Heart Circ Physiol 2010;299:H1255-1261.

-39 Frantz S, Adamek A, Fraccarollo D, Tillmanns J, Widder JD, Dienesch C, Schafer A, Podolskaya A, Held M, Ruetten H, Ertl G, Bauersachs J: The eNOS enhancer AVE 9488: a novel cardioprotectant against ischemia reperfusion injury. Basic Res Cardiol 2009;104:773-779.

40 Cheng Y, Jiang S, Hu R, Lv L: Potential mechanism for endothelial progenitor cell therapy in acute myocardial infarction: Activation of VEGF- PI3K/Akte-NOS pathway. Ann Clin Lab Sci 2013;43:395-401.

41 Lv L, Jiang SS, Xu J, Gong JB, Cheng Y: Protective effect of ligustrazine against myocardial ischaemia reperfusion in rats: the role of endothelial nitric oxide synthase. Clin Exp Pharmacol Physiol 2012;39:2027.

42 Li T, Yu J, Chen R, Wu J, Fei J, Bo Q, Xue L, Li D: Mycophenolate mofetil attenuates myocardial ischemiareperfusion injury via regulation of the TLR4/NF-kappaB signaling pathway. Pharmazie 2014;69:850-855.

-43 Moens AL, Claeys MJ, Timmermans JP, Vrints CJ: Myocardial ischemia/reperfusion-injury, a clinical view on a complex pathophysiological process. Int J Cardiol 2005;100:179-190. 


\section{Cellular Physiology Cell Physiol Biochem 2018;49:1646-1658

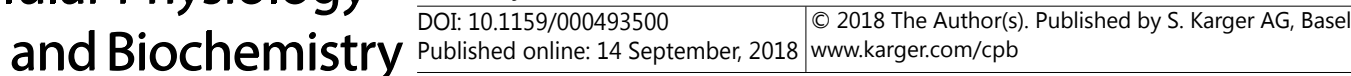

Huang et al.: Ophiopogonin D Reduces Myocardial I/R Injury via CYP2J3/EETs

44 Cai X, Wang X, Li J, Chen S: Protective effect of glycyrrhizin on myocardial ischemia/reperfusion injuryinduced oxidative stress, inducible nitric oxide synthase and inflammatory reactions through high-mobility group box 1 and mitogen-activated protein kinase expression. Exp Ther Med 2017;14:1219-1226.

45 Wang Y, Zhang ZZ, Wu Y, Zhan J, He XH, Wang YL: Honokiol protects rat hearts against myocardial ischemia reperfusion injury by reducing oxidative stress and inflammation. Exp Ther Med 2013;5:315-319.

-46 Zhang MQ, Zheng YL, Chen H, Tu JF, Shen Y, Guo JP, Yang XH, Yuan SR, Chen LZ, Chai JJ, Lu JH, Zhai CL: Sodium tanshinone IIA sulfonate protects rat myocardium against ischemia-reperfusion injury via activation of PI3K/Akt/FOXO3A/Bim pathway. Acta Pharmacol Sin 2013;34:1386-1396.

-47 Swales KE, Moore R, Truss NJ, Tucker A, Warner TD, Negishi M, Bishop-Bailey D: Pregnane X receptor regulates drug metabolism and transport in the vasculature and protects from oxidative stress. Cardiovasc Res 2012;93:674-681.

-48 Swales KE, Bishop-Bailey D: The potential use of the pregnane X receptor in cardiovascular therapy. Expert Rev Cardiovasc Ther 2012;10:1079-1082.

49 Zhou T, You WT, Ma ZC, Liang QD, Tan HL, Xiao CR, Tang XL, Zhang BL, Wang YG, Gao Y: Ginkgolide B protects human umbilical vein endothelial cells against xenobiotic injuries via PXR activation. Acta Pharmacol Sin 2016;37:177-186.

50 Zhu H, Chen Z, Ma Z, Tan H, Xiao C, Tang X, Zhang B, Wang Y, Gao Y: Tanshinone IIA Protects Endothelial Cells from H(2)O(2)-Induced Injuries via PXR Activation. Biomol Ther (Seoul) 2017;25:599-608.

-51 Zhang X, Wang Y, Ma Z, Liang Q, Tang X, Hu D, Tan H, Xiao C, Gao Y: Tanshinone IIA ameliorates dextran sulfate sodium-induced inflammatory bowel disease via the pregnane X receptor. Drug Des Devel Ther 2015;9:6343-6362. 